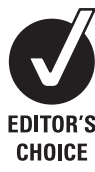

${ }^{1}$ Department of Emergency Medicine, Hairmyres Hospital, East Kilbride, UK; ${ }^{2}$ Department of Emergency Medicine, Glasgow Royal Infirmary, Glasgow, UK; ${ }^{3}$ Department of Emergency Medicine, Crosshouse Hospital,

Kilmarnock, UK; ${ }^{4}$ Department of Emergency Medicine, Royal Free Hospital, London, UK

Correspondence to:

Miss J M Vardy, Department of Emergency Medicine, Hairmyres Hospital, East Kilbride G75 8RG, UK; jenvardy@doctors.net.uk

Accepted 27 February 2008

\title{
Audit of the safety and effectiveness of ketamine for procedural sedation in the emergency department
}

\author{
J M Vardy, ${ }^{1}$ N Dignon, ${ }^{2}$ N Mukherjee, ${ }^{3}$ D M Sami, ${ }^{4}$ G Balachandran, ${ }^{2}$ S Taylor ${ }^{2}$
}

\begin{abstract}
Aim: To examine the effectiveness and safety of the sedative agents used in the emergency department following the introduction of ketamine as an agent for procedural sedation
\end{abstract}

Methods: A 2-year prospective audit of sedation practice was undertaken. This specifically examined the rationale behind a doctor's choice of sedative agent, the depth of sedation achieved, adverse events and the time taken to regain full orientation.

Results: 210 patients were included of whom 85 (40\%) were given ketamine, 107 (51\%) midazolam and 18 (9\%) propofol. The median time to full orientation was $25 \mathrm{~min}$ for ketamine, 30 min for midazolam and 10 min for propofol. Complications occurred in $15.9 \%$ of sedations overall (14.6\% of those given ketamine, $15.8 \%$ given midazolam and $22.2 \%$ given propofol). Apnoea and hypoxia most often occurred with midazolam and propofol, while hypertension and hypertonicity were encountered more frequently with ketamine. In addition, $19.5 \%$ of patients given ketamine suffered the reemergence phenomenon. The association between deep sedation with no response to pain and adverse events encountered with midazolam does not occur with ketamine.

Conclusions: Ketamine is both safe and effective and compares favourably with midazolam as an agent for procedural sedation in the emergency department. Although the re-emergence phenomenon occurred, no psychological sequelae were encountered after return to full orientation. Ketamine may be particularly useful in groups of patients at high risk of adverse effects with midazolam.

Ketamine produces a dissociative state and provides good analgesia at a subanaesthetic dosage. ${ }^{1}$ The American College of Emergency Physicians (ACEP) recognises that ketamine, midazolam, propofol, fentanyl and etomidate are all in common usage for procedural sedation within the emergency department (ED). However, their only level A recommendation favours ketamine in children. They give no recommendations for the use of ketamine in adults. ${ }^{2}$

Ketamine was recently introduced into the ED at the Glasgow Royal Infirmary for use in procedural sedation. Doctors follow departmental protocol, administering ketamine $0.5 \mathrm{mg} / \mathrm{kg}$ initially to a maximum of $1.0 \mathrm{mg} / \mathrm{kg}$ intravenously. The protocol does not include premedication with midazolam. Ketamine is contraindicated in patients with chronic or current hypertension (systolic blood pressure $>150 \mathrm{~mm} \mathrm{Hg}$ ) and in patients with a history of hallucinations, porphyria or pregnancy, and is avoided in head injured or alcoholic patients.
This audit was undertaken for the purpose of clinical governance to examine sedation practice within the department with particular focus on comparing the effectiveness and safety of newly introduced ketamine with the pharmacological agents previously available (midazolam and propofol).

\section{Studies comparing the incidence of adverse event with different sedation agents}

Adverse events are reported to occur in $4-18 \%$ of procedural sedations. The ACEP clinical policy document lists respiratory depression as the most concerning of adverse events, ${ }^{2}$ which may also include loss of a patent airway, hypotension, agitation, bradycardia, vomiting and myoclonia..$^{3-7}$ Fortunately, adverse events tend to be transient, mainly occur during the procedure and rarely affect patient disposition. ${ }^{458}$ The British National Formulary warns that extraneous muscle movements, cardiovascular stimulation with tachycardia and hypertension, hallucinations, nightmares and other transient psychotic effects may specifically occur with ketamine. ${ }^{1}$

\section{Recovery, recall and re-emergence phenomenon}

Available information suggests a recovery time to full orientation for ketamine which is similar to midazolam and longer than for propofol. ${ }^{4-11}$ Recall of the procedure is thought to be uncommon and its absence important for patient satisfaction, however some patients with recall will still be perfectly satisfied with their procedure. ${ }^{12}{ }^{13}$

Ketamine is said to cause distressing re-emergence phenomenon in adults. However, one review of 70 adult cases found that, although $25 \%$ recalled dreaming, less than a third found this unpleasant. This concurs with a study in patients aged 1621 years where none suffered hallucinations, delirium or other serious re-emergence reactions. ${ }^{514}$

\section{METHODS}

Data were collected from August 2005 to September 2007. Forms were left in the resuscitation area and filled in anonymously by the doctor performing the sedation. Doctors were encouraged to follow their normal practice and not be influenced by the audit process. All patients received high-flow oxygen and were monitored by continuous ECG, pulse oximetry and noninvasive blood pressure measurement.

The following data were recorded:

- age and sex of patient;

- time of admission and discharge;

- procedure performed; 
Table 1 Grade of doctor and agent used for sedation

\begin{tabular}{llllllc}
\hline & Consultant & SpR & Middle grade & SHO & Not stated & Total \\
\hline Ketamine & 48 & 6 & 29 & 0 & 2 & $85(40 \%)$ \\
Midazolam & 13 & 10 & 72 & 9 & 3 & $107(51 \%)$ \\
Propofol & 1 & 8 & 9 & 0 & 0 & $18(9 \%)$ \\
Total & $62(30 \%)$ & $24(11 \%)$ & $110(52 \%)$ & $9(4 \%)$ & $5(2 \%)$ & $210(100 \%)$ \\
\hline
\end{tabular}

- pharmacological agent(s) used, timing and dosage;

- why the pharmacological agent was selected;

- patient's response to the procedure (chosen from no response/response to pain/response to speech);

- time after sedation that the patient regained full orientation;

- if re-emergence phenomena of any description occurred;

- whether the patient could recall the procedure;

- adverse events recorded with a tick chart for recognised complications with additional free text space;

- patient disposal.

Data were analysed in Minitab 13 and graphs prepared in Microsoft Excel.

\section{RESULTS}

\section{Age of patients, reason for sedation}

The audit included 210 procedural sedations. Patients were aged between 11 and 103 years (mean 49 years) including eight children aged $\leqslant 16$ years; $53 \%$ of the patients were male. The reasons for sedation were reduction of a dislocation in $62 \%$, fracture manipulation in 34\% and "other procedures" including foreign body removal, dressing change, wound repair and abscess drainage in $3 \%$. The median time from arrival in the ED to sedation was 55 min (range 8-270) with no significant difference between daytime and night-time.

\section{Sedation agent used, grade of doctor and reason for selecting the agent}

Of the 210 sedations, ketamine was used as the primary sedation agent in $85(40 \%)$, midazolam in 107 (51\%) and propofol in 18 (9\%). The median dose of ketamine administered was $65 \mathrm{mg}$ (range 6-180 mg), the median dose of midazolam used was $5 \mathrm{mg}$ (range 2-20 mg) and the median dose of propofol administered was $100 \mathrm{mg}$ (range 20-200 mg).

In five procedural sedations ketamine $1 \mathrm{mg} / \mathrm{kg}$ (the maximum dose permitted by our protocol) did not produce sufficient sedation and a bolus of midazolam (4 cases) or propofol (1 case) was required. Likewise, one patient was insufficiently sedated after $13 \mathrm{mg}$ midazolam and was successfully given $100 \mathrm{mg}$ ketamine. A total of 206 procedures (98\%) were successful; all four failed procedures used midazolam.

In addition to sedation, $64 \%$ of patients were given opiate analgesia, $53 \%$ morphine and $17 \%$ fentanyl or alfentanil. This includes all opiates whether given prehospital, in the ED prior to radiography or before or during the actual procedure.
Table 1 shows the grade of doctor using each sedative agent and table 2 lists the reasons given for selecting a particular agent. Ketamine and propofol were significantly more likely to be selected on grounds of their pharmacological profile while midazolam was significantly more likely to be chosen because of its familiarity to the doctor ( $p<0.001, \chi^{2}$ test).

\section{Time to full orientation}

The median time taken for patients to recover full orientation was 25 min (range $0-155$ ). The median time to full orientation was significantly lower for propofol at $(10 \mathrm{~min})$ than for ketamine (25 min) and midazolam (30 min) $(p<0.001$, MannWhitney test). The time taken for $85 \%$ of patients to be fully orientated was $20 \mathrm{~min}$ for those sedated with propofol compared with $40 \mathrm{~min}$ for ketamine and $60 \mathrm{~min}$ with midazolam (fig 1).

\section{Recall}

Three patients (4\%) given ketamine, 22 (24\%) given midazolam and $1(6 \%)$ given propofol reported recall of the procedure. There was a significant association between midazolam and recall $\left(p<0.001, \chi^{2}\right.$ test $)$.

\section{Re-emergence phenomenon}

There was a significant association between ketamine and the re-emergence phenomenon, with 16 patients (19.5\%) given ketamine affected compared with $1(5.5 \%)$ given propofol and none of those given midazolam ( $p<0.001, \chi^{2}$ test) Patients suffering the re-emergence phenomenon with ketamine were significantly younger than those unaffected (30 vs 49 years, $\mathrm{p}=0.03$, Mann-Whitney test). No psychological symptoms persisted beyond recovery to full orientation and no patient required admission as a result of the re-emergence phenomenon.

\section{Adverse events}

In nine cases no data for adverse events were recorded. Analysis of the remaining 201 patients revealed that an adverse event occurred in $32(15.9 \%)$ procedural sedations. Hypoxia was defined as desaturation below $94 \%$ and hypertension was defined as a systolic blood pressure of $>150 \mathrm{~mm} \mathrm{Hg}$.

No significant association was found between any single sedative agent and adverse events ( $p=0.73, \chi^{2}$ test). No patient required admission as a consequence of an adverse event. The incidence of adverse events with each sedative agent is given in table 3 .

Table 2 Reasons for selecting an agent

\begin{tabular}{|c|c|c|c|c|c|c|}
\hline & Familiarity & $\begin{array}{l}\text { Pharmacological } \\
\text { profile }\end{array}$ & $\begin{array}{l}\text { Teaching } \\
\text { purposes }\end{array}$ & $\begin{array}{l}\text { Contraindication to } \\
\text { another agent }\end{array}$ & Not stated & Total \\
\hline Ketamine & 11 & 34 & 24 & 3 & 13 & 85 \\
\hline Midazolam & 66 & 13 & 1 & 18 & 9 & 107 \\
\hline Propofol & 1 & 14 & 1 & 2 & 0 & 18 \\
\hline Total & $78(37 \%)$ & $61(29 \%)$ & $26(12 \%)$ & $23(11 \%)$ & $22(10 \%)$ & 210 \\
\hline
\end{tabular}




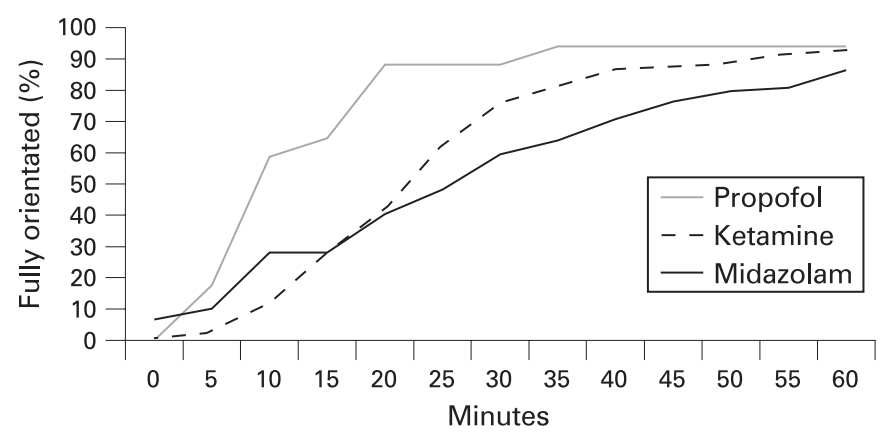

Figure 1 Recovery time to full orientation in the first hour after sedation.

\section{Level of sedation}

Significantly more patients were sedated to a level of "no response" with ketamine than with midazolam ( $<<0.001, \chi^{2}$ test, table 4). Patients with "no response" were significantly older on average than patients with "response to speech" (53 vs 41 years, $p=0.009$, one-way ANOVA).

Table 5 shows the incidence of adverse events at each level of sedation. The highest incidence occurred in the "no response" group but this was not statistically significant $(p=0.1)$. No relationship was found between the incidence of adverse events and the level of sedation with ketamine, but there was a significant increase in the incidence of adverse events when sedated to a level of "no response" with midazolam ( $p=0.001, \chi^{2}$ test).

\section{Relationship between age, recovery to full orientation and adverse events}

Patients suffering an adverse event took significantly longer to recover to full orientation (29.5 vs $22.5 \mathrm{~min}, \mathrm{p}=0.012$, MannWhitney test) and were significantly older than patients without an adverse event (55 vs 48 years, $p=0.047$, Mann-Whitney test) but had received a similar dose of sedation to those who did not (ketamine 60 vs $70 \mathrm{mg}, \mathrm{p}=0.41$, Mann-Whitney test; midazolam 5 vs $4 \mathrm{mg}, \mathrm{p}=0.26$, Mann-Whitney test).

Increasing age significantly lengthened the time of recovery to full orientation in patients given midazolam but not in patients given ketamine (midazolam, $p=0.02$, Mann-Whitney test, ketamine, $p=0.85$, Mann-Whitney test). This is despite the fact that the dose of midazolam decreased with age (Pearson's correlation $\mathrm{p}=0.032$ ).

\section{DISCUSSION}

\section{Effectiveness and safety of ketamine}

This audit was undertaken to examine the effectiveness and safety of procedural sedation in the ED as a whole, with particular emphasis on assessing the effectiveness and safety of ketamine. The effectiveness of ketamine was demonstrated by a
Table 4 Percentage of patients sedated to each level with each sedative agent

\begin{tabular}{llll}
\hline Level of sedation & $\begin{array}{l}\text { No } \\
\text { response (\%) }\end{array}$ & $\begin{array}{l}\text { Response to } \\
\text { pain (\%) }\end{array}$ & $\begin{array}{l}\text { Response to } \\
\text { speech (\%) }\end{array}$ \\
\hline Ketamine & 58 & 32 & 11 \\
Midazolam & 23 & 45 & 32 \\
Propofol & 50 & 39 & 11 \\
Total for all agents & 37 & 40 & 23 \\
\hline
\end{tabular}

$100 \%$ procedural success rate. Safety was comparable to other agents in use with $14.6 \%$ of patients given ketamine suffering an adverse event compared with $15.9 \%$ overall.

\section{Adverse events}

The incidence of adverse events in this audit is at the higher end of that found in the literature, possibly reflecting a low threshold for our definition of an adverse event. It is reassuring that no patient required admission as a result.

The incidence of adverse events was comparable for ketamine and midazolam at $14.6 \%$ and $15.8 \%$, respectively. The numbers were too small to attain statistical significance, but it appears that both apnoea and hypoxia-arguably the most serious adverse events observed-and also hypotension are associated with midazolam. Hypertonicity and hypertension were associated with ketamine, reiterating the importance of careful patient selection to exclude patients with a predisposition to hypertension from ketamine sedation. Although the numbers were small, it is concerning that $17 \%$ suffered apnoea or hypoxia with propofol, probably reflecting the narrower margin of safety with this sedation agent.

\section{Time to full orientation}

Overall time of recovery to full orientation was similar for ketamine and midazolam. However, older patients given midazolam had a significantly longer recovery time to full orientation than younger patients despite receiving lower doses of sedative. This was not a feature of ketamine. This is important as patients who took longer to recover to full orientation were more likely to suffer an adverse event.

\section{Level of sedation}

Of those given midazolam, $68 \%$ were sedated to a level of responding to pain or lower which some consider a level of sedation equivalent to a general anaesthetic. Patients given midazolam had a significantly higher incidence of adverse events if they were sedated to a level of "no response", whereas patients given ketamine demonstrated a more equal incidence at each level of sedation. This difference may be explained by a difference in pharmacological profiles, with ketamine able to produce a state of dissociative analgesia.

Table 3 Percentage of patients suffering adverse events and the agent used

\begin{tabular}{lcccc}
\hline Adverse event & Ketamine (\%) & Midazolam (\%) & Propofol (\%) & Overall (\%) \\
\hline Apnoea or hypoxia & 1 & 12 & 17 & 8 \\
Hypertonicity & 7 & 0 & 6 & 3 \\
Hypertension & 5 & 0 & 0 & 2 \\
Hypotension & 0 & 3 & 0 & 1 \\
Vomiting & 1 & 1 & 0 & 1 \\
Complication rate & 14.6 & 15.8 & 22.2 & 15.9 \\
\hline
\end{tabular}


Table 5 Percentage of adverse events occurring at each level of sedation with each sedative agent

\begin{tabular}{llll}
\hline Level of sedation & $\begin{array}{l}\text { No } \\
\text { response (\%) }\end{array}$ & $\begin{array}{l}\text { Response to } \\
\text { pain (\%) }\end{array}$ & $\begin{array}{l}\text { Response to } \\
\text { speech (\%) }\end{array}$ \\
\hline Ketamine & 18 & 14 & 17 \\
Midazolam & 41 & 11 & 6 \\
Propofol & 0 & 57 & 0 \\
$\begin{array}{l}\text { Overall adverse } \\
\text { events }\end{array}$ & 23 & 16 & 7 \\
\hline
\end{tabular}

\section{Re-emergence phenomenon and recall}

Re-emergence phenomenon occurred in one-fifth of patients given ketamine, predominantly in younger patients. The reemergence phenomenon most commonly described by patients was a vivid dream that was unusual but not disturbing. Nightmares that were frightening or unpleasant were described less often. One example is of an 11-year-old patient who dreamt she was on a train with a dead man, possibly influenced by a cardiac arrest that arrived in the neighbouring cubicle after she was sedated. Once fully orientated she told staff that she had had a bad dream and understood it had not been real.

Many clinicians are concerned about this well publicised adverse effect of ketamine. The possibility of its occurrence must be taken into account when choosing a safe sedation agent; however, with careful patient selection and appropriate environmental and medical management, the re-emergence phenomenon can be regarded as a manageable aspect of the pharmacological profile of ketamine.

\section{Limitations of the study}

There were two major limitations. First, the analysis of propofol data was limited as patient numbers were too small to meaningfully interpret if any subdivision was required. Second, while subjectively we felt that the re-emergence phenomenon impacted greatly on staff satisfaction, this was felt to be less important to patients than experiencing pain or having recall of the procedure. We plan to examine this in the ongoing audit of sedation practice within the department.

\section{CONCLUSIONS}

Ketamine is safe and effective and shown to have at least comparable safety and effectiveness to midazolam. The successful introduction of ketamine into our ED was greatly assisted by the presence of clear departmental guidelines. As a result of this audit, departmental guidelines for the use of midazolam have been created and the impact of this will be assessed through ongoing audit of procedural sedation.
Apnoea and hypoxia are considered the most concerning complications by the American College of Emergency Physicians. We argue that ketamine is safer than midazolam and propofol as the incidence of apnoea or hypoxia was only $1 \%$ with ketamine compared with $12 \%$ with midazolam and $17 \%$ with propofol. Midazolam was also associated with a significant incidence of adverse events associated with oversedation.

In the ED of Glasgow Royal Infirmary we were accustomed to using midazolam and were instinctively wary of features of sedation known to be negatively prognostic with it. Ketamine has a different mechanism of action and it will take time to become familiar and comfortable with its use. This is an effort worth making, as ketamine is both safe and effective for procedural sedation in the ED and may be particularly useful in patients such as the elderly who are at greater risk of adverse events with midazolam.

Funding: None.

Competing interests: None.

\section{REFERENCES}

1. Anon. British National Formulary. Edition 53. British Medical Association and Royal Pharmaceutical Society. www.bnf.org/bnf (accessed 4 July 2007).

2. Godwin SA, Caro DA, Wolf SJ, et al. American College of Emergency Physicians. Clinical policy: procedural sedation and analgesia in the emergency department. Ann Emerg Med 2005;45:177-96.

3. Mensour M, Pineau R, Sahai V, et al. Emergency department procedural sedation and analgesia: a Canadian Community Effectiveness and Safety Study (ACCESS). Can J Emerg Med Care 2006;8:94-9.

4. Duncan RA, Symington L, Thakore S. Sedation practice in a Scottish teaching hospital emergency department. Emerg Med J 2006;23:684-6.

5. Chudnofsky C, Weber J, Stoyanoff P, et al. A combination of midazolam and ketamine for procedural sedation and analgesia in adult emergency department patients. Acad Emerg Med 2000;7:228-35.

6. Frymann SJ, Cumberbatch GLA, Stearman ASL. Reduction of dislocated hip prosthesis in the emergency department using conscious sedation: a prospective study. Emerg Med J 2005;22:807-9.

7. Hogan K, Sacchetti A, Aman L, et al. The safety of single-physician procedural sedation in the emergency department. Emerg Med J 2006;23:922-3.

8. Newman DH, Azer MM, Pitetti RD, et al. When is a patient safe for discharge after procedural sedation? The timing of adverse effect events in 1367 paediatric procedural sedations. Ann Emerg Med 2003;42:627-35.

9. Havel CJ, Strait RT, Hennes H. A clinical trial of propofol vs midazolam for procedural sedation in a pediatric emergency department. Acad Emerg Med 1999;6:989-97.

10. Taylor D, O'Brien D, Ritchie P, et al. Propofol versus midazolam/fentanyl for reduction of anterior shoulder dislocation. Acad Emerg Med 2005;12:13-9.

11. Dunn T, Mossop D, Newton A, et al. Propofol for procedural sedation in the emergency department. Emerg Med J 2007;24:459.

12. Dunn MJG, Mitchell R, Souza CD, et al. Evaluation of propofol and remifentanil for intravenous sedation for reducing shoulder dislocations in the emergency department. Emerg Med J 2006;23:57-8.

13. Swann A, Williams J, Fatovich D. Recall after procedural sedation in the emergency department. Emerg Med J 2007;24:322-4.

14. Green SM, Sherwin TS. Incidence and severity of recovery agitation after ketamine sedation in young adults. Am J Emerg Med 2005;23:142-4. 